\title{
Plataforma on-line para apoio à tomada de decisão e planejamento de segurança para mulheres vivendo com violência doméstica no Brasil: bases teórico- metodológicas e resultados preliminares
}

\author{
Online-based platform to support decision-making and safety planning for women living with \\ domestic violence in Brazil: theoretical and methodological framework and preliminary results
}

\author{
Vera Lucia Costa da Silva ${ }^{1}$ \\ Sandra Marques Prado \\ Marcos Claudio Signorelli ${ }^{3}$
}

\begin{abstract}
Resumo
Esta pesquisa é inspirada em projetos similares desenvolvidos e testados internacionalmente, cujos resultados demonstraram que mulheres em situação de violência por parceiro íntimo (VPI) sentiram-se apoiadas em suas decisões e menos conflitos decisórios sobre sua segurança na relação afetivo-conjugal após o uso de plataformas de apoio on-line. Com base nas experiências internacionais objetivamos descrever as bases teórico-metodológicas e analisar preliminarmente a viabilidade de uma plataforma on-line de apoio às mulheres em situação de VPI adaptada ao contexto brasileiro. O estudo vem sendo desenvolvido desde janeiro/2018 junto à Casa da Mulher Brasileira de Curitiba (CMBC), por meio de abordagem qualitativa de pesquisa baseada em pressupostos da pesquisa ação-participativa, observação participante e entrevistas semiestruturadas com profissionais e usuárias da CMBC. Os resultados preliminares evidenciaram que a maioria das participantes consideram viável a proposta, destacando desafios e potencialidades: 1) agilidade, segurança e anonimidade oferecida por ferramentas on-line, evitando a exposição das usuárias; 2) a utilização de um recurso on-line como primeiro passo em direção às denúncias formais, auxiliando a conexão com a rede de serviços; 3) o reconhecimento de situações de abuso que podem ser fomentadas por meio da disseminação de informações sobre violência e também de políticas públicas preventivas; 4) a necessidade de ponderar a respeito da segurança das usuárias da possível plataforma, pois poderia ser flagrada pelo agressor utilizando-a, o que poderia gerar mais violência. Os resultados preliminares vêm auxiliando no delineamento de uma versão brasileira para a plataforma, culturalmente apropriada e que atenda as características das mulheres brasileiras.
\end{abstract}

Palavras-chave: Plataforma on-line. Violência doméstica. Violência por parceiro íntimo. Políticas Públicas.

\section{Abstract}

This study is inspired by similar researches developed and tested internationally. These researches showed that women living with intimate partner violence (IPV) felt supported in their decisions and

\footnotetext{
1 Mestranda em Desenvolvimento Territorial Sustentável pela Universidade Federal do Paraná (PPGDTS/UFPR). Professora de Serviço Social na Faculdade Educacional da Lapa/FAEL. E-mail: veraluciaconsultoria@ yahoo.com.br

${ }^{2}$ Diretora da Casa da Mulher Brasileira de Curitiba, Paraná, Brasil. Pós-Graduada em Ciência Política pelo IBEX. Email: sprado@cmb.curitiba.pr.gov.br

${ }^{3}$ Doutor em Saúde Coletiva pela Universidade Federal de São Paulo/Escola Paulista de Medicina - UNIFESP. Professor dos Programas de Pós-Graduação em Desenvolvimento Territorial Sustentável e Saúde Coletiva da UFPR. E-mail: signorelli.marcos@gmail.com
} 
fewer decisional conflict about their safety in intimate relationships after using these online-based support platforms. Based on these international experiences we aim to describe the theoretical and methodological framework and to analyse the viability of an online platform to support women in situation of IPV adapted to the Brazilian context, whose preliminary results are discussed in this paper. The study has been conducted since January/2018 at the House of the Brazilian Woman of Curitiba (HBWC). We adopted qualitative approaches based on principles of participatory-action research, participant observation and semi-structured interviews with professionals and users of HBWC. Preliminary results showed that the majority of participants consider the proposal feasible, highlighting challenges and potentials: 1) agility, safety and anonymity provided by online tools, avoiding user's exposure; 2) the use of an online resource as a first step towards formal complaints, helping to link them with the service network; 3) the recognition of situations of abuse that can be fostered through the dissemination of information about violence and also through preventive public policies; 4) the need to consider the safety of users, as they could be caught by the aggressors using it, which could generate more violence. Preliminary results have been helping to design a culturally appropriate Brazilian version of the platform that meets the characteristics of Brazilian women.

Keywords: Online-based platform. Domestic violence. Intimate partner violence. Public policies.

\section{Introdução}

A violência contra a mulher é um problema de saúde pública e que afeta 1 (uma) em cada 3 (três) mulheres no mundo, conforme dados da Organização Mundial da Saúde (OMS), publicado no Relatório Mundial sobre Violência e Saúde (KRUG et al., 2002, p. 92). Ainda, uma das formas mais comuns de violência contra as mulheres é a praticada pelo marido, companheiro(a) ou um(a) parceiro íntimo(a) ou ex, sendo denominada Violência por Parceiro Íntimo (VPI).

Para a mulher, viver em um relacionamento abusivo e violento deixa profundas consequências que afetam a questão da saúde, da felicidade, do bem-estar e do senso de autoestima, além de sua capacidade de participar no mundo.

No que tange aos impactos na saúde de uma mulher acometida pela VPI, a violência tem sido vinculada a uma série de diferentes resultados em saúde, tanto imediatos quanto em longo prazo. Segundo estudos, "com relação às consequências diretas na saúde, tais como lesões, ser uma vítima da violência também aumenta o risco de uma mulher vir a ter uma saúde precária no futuro" (KRUG et al., 2002, p. 102).

Quanto às consequências em longo prazo, as mulheres em situação de VPI estão mais suscetíveis ao uso do tabaco e do álcool, portanto, ser uma vítima de violência pode ser considerado como um fator de risco para diversas doenças e graves problemas de saúde. Haja vista, encontram dificuldades para cuidar de si mesmas e de seus filhos, muitas vezes ainda encontram limitações para conseguir procurar empregos e seguir carreiras. 
Para Clemente, Martinez e Hoffmann-Horochovski (2018, p.49):

Falar em violência contra as mulheres é falar do ideário do acesso sistemático dos homens sobre seus corpos. A naturalização patriarcal opera com mecanismos adaptados e atualizados que são introjetados e reproduzidos, gerando comportamentos sociais que banalizam a violência contra a mulher.

Nesse cenário, independente da classe social, da condição socioeconômica, da religião ou cultura, a VPI ocorre em todos os países, ou seja, a violência contra a mulher é considerada como consequência da subalternidade [...] proposta por meio da naturalização patriarcal (CLEMENTE; MARTINEZ; HOFFMANN-HOROCHOVKSI, 2018. p. 45).

Com base nos dados extraídos do Monitor da Violência (2017), lançado pelo Núcleo de Estudos da Violência (NEV-USP), revela-se que doze mulheres são assassinadas todos os dias, em média, no Brasil. Ou seja, casos de mulheres mortas em crimes de ódio motivados pela condição de gênero. Uma mulher é assassinada a cada duas horas no Brasil, taxa de 4,3 mortes para cada grupo de 100 mil pessoas do sexo feminino. Conforme dados da Organização Mundial da Saúde (OMS), publicado no Relatório Mundial sobre Violência e Saúde (2002, p. 92), estima-se que no mundo, uma em cada três mulheres é, já foi ou será vítima de violência doméstica.

No que tange às relações de gênero e violência, toma-se como base Acosta et al. (2015, p. 122), que relatam que o número de mulheres vitimadas ainda é elevado, e que os registros apontam como sendo majoritariamente o agressor o próprio marido ou companheiro; mais de $40 \%$ das violências resultam em lesões corporais em consequência de socos, tapas, chutes, queimaduras e espancamentos. No entanto, a magnitude do problema é bem maior, pois o silêncio de muitas vítimas faz com que inúmeros casos não sejam denunciados, e isso mascara os dados epidemiológicos.

Nessa direção, a presente pesquisa é inspirada em projetos similares previamente desenvolvidos e testados nos EUA - Iris e MyPlan App (EDEN et al., 2015), no Canadá - iCan (FORD-GILBOE et al., 2017), na Austrália - iDecide (HEGARTY et al., 2015; 2019; TARZIA et al, 2016). Para testar a eficácia desse projeto na Australia, as autoras supracitadas utilizaram da metodologia do ensaio clínico randomizado com mulheres entre 16 e 50 anos de idade residentes na Austrália, as quais já foram acometidas por VPI e que tinham acesso seguro a um computador e conexão à Internet. Os resultados qualitativos desse projeto indicaram que algumas mulheres acham que uma ferramenta on-line é uma fonte útil de motivação e de apoio (HEGARTY, 2015; 2019).

$\mathrm{Na}$ Nova Zelândia, foi desenvolvido o iSafe (KOZIOL-MCLAIN et al., 2015), cujos resultados demonstraram que as mulheres em situação de violência doméstica (VD) sentiram-se apoiadas em suas decisões, aumentaram a tomada ativa de decisão e sentiram menos conflitos 
decisórios sobre sua segurança na relação após o uso de plataformas de apoio on-line, considerando, então, como a justificativa desta pesquisa.

Conforme Kelsey Hegarty et al. (2019), ao publicar a avaliação da plataforma on-line “iDecide", os resultados qualitativos indicaram que as participantes acharam a intervenção de apoio uma motivação para a ação e, ainda, algumas mulheres vítimas de violência compreenderam que uma ferramenta on-line é uma fonte útil de motivação e apoio, sobretudo quanto ao fator tomada de decisão e de planejamento de segurança.

Segundo Nancy Glass et al. (2016), autoras das plataformas on-line testadas nos Estados Unidos (Iris e MyPlan), as mulheres vítimas de abuso enfrentam decisões de segurança bastante complexas, perigosas e difíceis. No entanto, a pedra angular das intervenções de VPI é o planejamento de segurança, que por sua vez é um processo dialógico que apoia a tomada de decisões de mulheres vítimas de abuso.

No que tange ao planejamento de segurança, considera-se fator primordial atender prioridades das mulheres acerca da tomada de decisão e no conflito decisório, por exemplo. Nessa direção, a partir da proposta aqui apresentada de uma "Plataforma on-line para apoio à tomada de decisão e planejamento de segurança para mulheres vivendo com violência doméstica no Brasil”, vislumbra-se a construção de mais um mecanismo em atenção às mulheres em situação de VPI no que tange a pensar em um plano para deixar ou permanecer no relacionamento abusivo.

Ressalta-se que embora existam no Brasil muitas políticas públicas (PPs) de atenção às mulheres em situação de violência doméstica, os dados de feminicídio ainda são alarmantes e isso sugere lacunas na implementação de PPs, bem como necessidade de novas práticas sustentáveis e com abrangência nacional. Dessa forma, a proposta de análise da viabilidade da plataforma on-line de planejamento de segurança no Brasil consiste em trabalhar com a mulher em situação de VPI para ela que possa identificar como pode agir para manter-se mais segura diante de novos atos de violência, ajudando-a na tomada de decisão, na identificação de uma rede de apoio socioassistencial e, sobretudo, no processo de empoderamento. Espera-se que ela mesma possa encontrar as respostas com apoio da plataforma: Será que devo deixar ou permanecer no relacionamento abusivo? Caso escolha deixar, que passos posso tomar para planejar com segurança a saída do relacionamento? Quanto risco estou correndo se decidir permanecer no relacionamento?

Este estudo baseia-se teoricamente na proposta de empoderamento de mulheres em situação de violência, conforme Mary Ann Dutton (1992). Ela utilizou um modelo de avaliação psicológica e intervenção para empoderamento das mulheres agredidas, tendo como objetivo aumentar a segurança 
da mulher agredida, ajudar a empoderá-la através da tomada de decisão e a curar o trauma psicológico do abuso. Contudo, há de se levar em conta por todos os profissionais as interpretações, pois muitas vezes as mulheres são mal interpretadas, como por exemplo, "quando a mulher não deixa a relação abusiva é porque não se sente motivada para proteger e garantir a sobrevivência de si mesma e de seus filhos na relação abusiva" (DUTTON, 1992, p. 49).

Por conseguinte, utilizou-se o modelo de empoderamento de Dutton (1992), que é doutora e pesquisadora nos Estados Unidos, fundadora do programa de Violência Familiar, um programa de pesquisa clínica aplicada que oferece serviços a vítimas e perpetradores de violência doméstica. Com relação ao modelo de empoderamento de Dutton (1992), ele se concentra na redução do conflito decisório das mulheres e no aumento do planejamento e do comportamento de segurança, uma iniciativa ainda muito pouco utilizada no cenário brasileiro.

Ainda, ressalta-se que os estudos relativos às plataformas já desenvolvidas nos outros países já citados tiveram como base também o modelo de empoderamento de Dutton (1992), construído a partir do método de avaliação psicológica e de intervenção testado pela referida autora em pesquisas realizadas com as mulheres vítimas de violência publicado em sua obra Empowering and healing the battered woman: a model for assessment and intervention.

O fenômeno da VPI no cenário brasileiro afeta cada vez mais parcelas do público feminino, que em muitos casos sofrem caladas, vítimas das mais perversas formas de violência. Não denunciam seus parceiros íntimos por motivos de ordem familiar como a proteção dos filhos, a afetividade, a questão econômica e até mesmo por temer novas violências. Ainda, soma-se a isso questões de segurança pessoal e risco social a que pode expor-se após a denúncia.

Nessa direção, o referido estudo baseia-se em estratégia promissora e recomendada internacionalmente para apoiar mulheres vítimas de VPI, que é o planejamento de segurança (do inglês safety planning). Esse planejamento visa auxiliar a mulher a identificar situações de abuso e como ela pode agir para se manter segura diante de novos atos de VPI ou perante a iminência desta. O plano deve ser específico ao contexto/caso, ajudando a aumentar a autonomia da mulher e sua capacidade de cuidar de si mesma (BAKER et al., 2004, p. 89).

Esse projeto é pioneiro no Brasil e objetiva analisar a viabilidade e particularidades de uma plataforma on-line de apoio às mulheres em situação de VPI, a qual será adaptada ao contexto brasileiro visando à redução do conflito decisório e apoiando o processo de tomada de decisão de largar uma relação abusiva. 
Para Simone de Beauvoir (1949, p. 10), "a mulher determina-se e diferencia-se em relação ao homem e não este em relação a ela; a fêmea é o inessencial perante o essencial”. Juntamente com Beauvoir, através de sua obra "O Segundo Sexo", reflete-se até os dias atuais qual a posição e o papel da mulher no mundo - ainda relegada a uma posição secundária, ou seja, a mulher assume um papel de coadjuvante na história. E esse papel muitas vezes legitima homens a agredirem suas parceiras.

Com intuito de problematizar esse cenário acerca da violência contra a mulher e o papel da mulher na sociedade historicamente construído, buscou-se a aproximação com teorias do "movimento feminista", que nasceu da luta das mulheres por um tratamento social igual ao que os homens recebem. Seu principal objetivo foi o de alcançar a igualdade entre os gêneros (homem e mulher), garantindo a participação ativa de mulheres nas realidades da sociedade onde vivem. Para a autora Joana Maria Pedro (2005), uma estudiosa sobre "relações de gênero", na perspectiva da "Teoria Feminista" o uso da palavra "gênero" tem uma história que é tributária de movimentos sociais de mulheres, feministas, gays e lésbicas. Tem uma trajetória que acompanha a luta por direitos civis, direitos humanos, enfim, igualdade e respeito (PEDRO, 2005, p. 78).

A partir dessa descrição do quadro teórico que embasa a proposta, propôs-se como objetivo para o referido projeto de pesquisa a "análise da viabilidade de plataforma on-line para apoio à tomada e decisão e planejamento de segurança para mulheres vivendo com violência doméstica no Brasil”. Na próxima seção do texto serão discutidos resultados preliminares da proposta, que vem sendo materializada com a participação de interlocutoras junto à Casa da Mulher Brasileira de Curitiba (CMBC), uma instituição especializada no atendimento de mulheres em situação de violência doméstica.

\section{Desenvolvimento}

Como um dos pontos de partida para a referida pesquisa, inicialmente, optou-se por um mapeamento e estudo aprofundado de plataformas similares desenvolvidas em outros países, incluindo os USA (Iris e MyPlan), Canadá (iCan), Austrália (iDecide) e Nova Zelândia (iSafe). Propôs-se como ferramenta investigativa um protocolo para delineamento e estudo da viabilidade da versão da plataforma on-line para o Brasil, de acordo com as demandas das mulheres brasileiras.

O trabalho vem sendo desenvolvido a partir da pesquisa qualitativa, utilizando como ponto de partida em relação ao referencial teórico-metodológico a autora Maria Cecília Minayo (2006, p. 84). 
Para ela, a pesquisa qualitativa contribui para que possamos compreender as relações, o ponto de vista e até mesmo o julgamento dos diferentes atores sobre a intervenção na qual participam, entendendo que suas vivências e reações fazem parte da construção da intervenção e também dos resultados. A referida autora conceitua a pesquisa qualitativa da seguinte forma:

Trabalha-se com atitudes, crenças, comportamentos e ações, procurando-se entender a forma como as pessoas interpretam e conferem sentido a suas experiências e ao mundo em que vivem. [...] compreender as relações, as visões e o julgamento dos diferentes atores sobre a intervenção na qual participam, entendendo que suas vivências e reações fazem parte da construção da intervenção e de seus resultados. Ou seja, acredita-se que exista uma relação dinâmica e inseparável entre o mundo real e a subjetividade dos participantes (MINAYO, 2006, p. 82).

Escolheu-se a abordagem qualitativa na referida pesquisa por compreender que tal metodologia contribui para a leitura de questões subjetivas e muito particulares dos atores sociais envolvidos na realidade investigada, e isso não pode ser quantificado. Ou seja, a abordagem qualitativa possibilita trabalhar com o universo de significados, motivos, aspirações, crenças, valores e atitudes, o que corresponde a um espaço mais profundo das relações sociais dos processos acerca do fenômeno violência contra a mulher.

Nesse sentido, a pesquisa de campo foi escolhida como ferramenta para elucidar embates interpretativos e reflexivos, sobretudo no que tange à construção coletiva de conhecimento, dados e de informações que vem servindo de subsídio para responder à questão norteadora: No Brasil, é viável a implementação de plataforma on-line de planejamento de segurança e apoio à tomada de decisão às mulheres vivendo em situação de violência, utilizada e testada em outros países?

Para embasar teoricamente a referida pesquisa optou-se tanto pela teoria do empoderamento de mulheres em situação de violência (DUTTON, 1992) quanto da teoria feminista. Segundo a autora Daniela Auad (2003), o feminismo é o: "movimento formado por mulheres críticas e questionadoras, nada têm a ver com o modelo de passividade e submissão [...]. Formam um grupo que de diferentes formas critica radicalmente o sistema econômico, político e social e que vivemos" (AUAD, 2003, p. 14).

Essa teoria visa corroborar com a produção do conhecimento por meio da captura de experiências vividas e de um novo (re)significado a partir do ponto de vista dos atores sociais, público-alvo participante da pesquisa. 
Como forma de investigação, adotou-se pressupostos da pesquisa-ação participativa (LIAMPUTTONG; EZZY, 2005), em que se inicia com um reconhecimento da realidade a ser pesquisada. Ou seja, o reconhecimento é uma análise situacional que produz ampla visão do contexto da pesquisa-ação, práticas atuais, dos participantes e envolvidos (DAVID TRIPP, 2005, p.453). Nessa dinâmica e para compreender a visão dos atores sociais no que tange à viabilidade da plataforma para o Brasil, na pesquisa de campo utilizou-se a técnica da entrevista com a participação ativa de profissionais e mulheres atendidas na Casa da Mulher Brasileira de Curitiba (CMBC), entre janeiro de 2018 até setembro de 2019.

Quanto às questões éticas, ressalta-se que a pesquisa seguiu todas as diretrizes nacionais e internacionais de pesquisa com seres humanos. Foi submetida aos Comitês de Ética em Pesquisas (CEP) com seres humanos da Universidade Federal do Paraná - Setor de Ciências da Saúde - e da Prefeitura Municipal de Curitiba. A pesquisa foi aprovada em ambos os CEP, seguindo a Resolução 466/2012, do Conselho Nacional de Saúde, e sua aprovação consta no parecer número CAAE 89411818.4.000.0102, registrada na Plataforma Brasil.

A partir de reuniões de planejamento com as equipes e gestoras da CMBC, optou-se por um roteiro de entrevista semiestruturada para entender, a partir da visão das(os) profissionais e das mulheres em situação de VPI, a respeito da viabilidade de implantação na versão brasileira de uma plataforma on-line de planejamento de segurança em atenção às mulheres em situação de VPI.

Após a aprovação do Comitê de Ética em Pesquisa da Universidade Federal do Paraná, iniciou-se a aplicação das entrevistas com profissionais que atuam, direta e/o ou indiretamente com mulheres em situação de violência atendidas na CMBC, e também com amostra de mulheres que consentiram em participar.

Participaram da pesquisa 28 pessoas com mais de 18 anos, que consentiram e assinaram o Termo de Consentimento Livre e Esclarecido (TCLE) da pesquisa, incluindo: profissionais (de segurança pública, do atendimento biopsicossocial, administrativo, Patrulha Maria da Penha, recepção e gestoras) e mulheres atendidas na CMBC.

Os dados foram transcritos e tabulados, adotando-se a "Análise de Conteúdo" à luz da técnica da autora Maria Laura Pubis Franco (2012). Para ela, a fala dos atores sociais partícipes da pesquisa assume a representação social no dinamismo interacional que se estabelece entre linguagem, pensamento e ação. Ou seja, essa abordagem contribuiu para dar um (re)significado e sentido ao que se propôs pesquisar, escutando-se as percepções dos(as) participantes em relação ao objeto de estudo, ouvindo suas sugestões e demandas para que possam posteriormente ser 
incorporadas nas etapas seguintes de desenvolvimento da "Plataforma On-line de Planejamento de Segurança" às mulheres em situação de VPI.

Com base em diálogos e entrevistas realizadas com os(as) profissionais da CMBC e mulheres em VPI, e a partir da sistematização e análise dos dados, constatou-se:

Dentre as 28 participantes, 25 consideraram viável a proposta da plataforma on-line para a realidade brasileira, realizando apontamentos importantes para seu processo de materialização, tais como:

1) a plataforma on-line pode oferecer agilidade, segurança e anonimidade, especialmente para mulheres que não queiram ficar expostas e que por medo ou vergonha deixam de procurar os serviços especializados presenciais, como delegacias especializadas ou a própria CMBC;

2) um recurso on-line pode servir de apoio também para profissionais da área que atendem mulheres em situação de VPI, que podem consultar informações e prestar um atendimento mais personalizado e humanizado;

3) a plataforma tem o potencial de se configurar como um primeiro canal de apoio à mulher, como "uma forma de desabafo e de tomar a decisão de denunciar o agressor", ajudando-a a dar o primeiro passo em direção a denúncias formais;

4) o aplicativo pode ser uma ferramenta didática, orientando o "passo a passo para que as mulheres se organizem e sejam encorajadas a fazer a denúncia, informando os locais apropriados para isso";

5) tem o potencial de ajudar a mulher a se reconhecer e se identificar como vivendo em uma situação de abuso, pois muitas não têm essa clareza. Ao responder perguntas sobre relações abusivas isso pode despertar percepções subjetivas sobre violência, que outrora poderiam passar despercebidas e naturalizadas;

6) a estratégia contribui com a rapidez na disseminação de informações sobre violência e possibilidade de conexão com os serviços de atendimento, facilitando a vida das mulheres. Também pode ser um diferencial na vida de mulheres que moram em áreas rurais ou locais com pouca disponibilidade de serviços presenciais da rede de atendimento.

Das(os) 3 participantes que não consideraram a plataforma on-line viável, os principais argumentos contrários foram:

1) em relação à segurança da usuária da plataforma, pois poderia ser um risco caso a mulher seja flagrada pelo agressor utilizando a ferramenta, e isso poderia resultar em mais agressões ou inclusive "quebrando o seu celular", aumentando o risco para a usuária; 
2) o preparo de quem receberá a denúncia, caso seja interligado com a rede de apoio presencial. É preciso que a rede de apoio presencial esteja preparada para receber essas mulheres, uma vez que a plataforma irá gerar uma demanda aos serviços presenciais;

3) uma das limitações de plataformas on-line para o cenário brasileiro é que muitas mulheres não têm acesso à internet, em regiões de zona rural por exemplo (sem cobertura de sinal) e outras que não possuem celular e/ou computador, especialmente as mais pobres. Há ainda as que possuem celular, mas não possuem créditos para utilizar a internet. Então foram sugeridas medidas alternativas, tais como a disponibilidade de baixar o aplicativo quando tiver wi-fi disponível e utilizar posteriormente suas funções no modo off-line.

Outras sugestões foram apontadas pelas interlocutoras, consideradas relevantes para a viabilidade da plataforma on-line de planejamento de segurança às mulheres vivendo com VPI no Brasil:

1) a plataforma deve ser discreta para não chamar atenção dos agressores, devendo ser o mais simples possível e de fácil acesso, com passo a passo e autoexplicativo, facilitando o uso para mulheres com baixa escolaridade ou com deficiência;

2) deve conter um click para conectar o aplicativo com a rede de proteção e telefones de parentes para apoio emergencial;

3) foi considerado fundamental projetar mecanismos de segurança e discrição em relação ao agressor, pois muitos monitoram os celulares das mulheres;

4) deve conter campos para que a mulher possa relatar o ocorrido, por áudio ou escrita, mas também uma estratégia semelhante a um "botão do pânico" para situações iminentes de violência grave e/ou feminicídio, ou ainda uma saída rápida da tela caso o agressor perceba a utilização, direcionando para outro assunto/tela, visando disfarçar o uso;

5) comunicação fácil, conectando-se com órgãos competentes que atendam as mulheres imediatamente;

6) oferecer a possibilidade de identificação e localização da vítima, especialmente caso a Patrulha Maria da Penha necessite ser acionada, o que poderia salvar vidas;

7) a plataforma deve conter informações sobre os tipos de violência e a Lei Maria da Penha, pois muitas não consideram violência psicológica como um tipo de violência;

8) a possibilidade de inserção de campos para identificação do agressor;

9) o ícone da plataforma on-line deve ser oculto de forma que o agressor não o identifique como um mecanismo para atender mulheres vítimas de violência. 
O Brasil apresenta inúmeros avanços no tocante às políticas públicas em atenção às mulheres vítimas de violência, como por exemplo, a constituição de um aparato legal conhecido como a Lei Maria da Penha, Lei no 11.340/2006, consagrando direitos e instituição de um sistema de proteção social às mulheres vítimas de violência, a qual visa também coibir a violência doméstica e familiar contra a mulher, assim como visa punir o agressor. O Artigo 20 da referida lei assegura às mulheres oportunidades para viver sem violência, preservar sua saúde física e mental, seu aperfeiçoamento moral, intelectual e social, independente de classe, raça, etnia e orientação sexual.

Em consonância com a Lei Maria da Penha e para maior compreensão dos tipos de violência que acometem as mulheres no Brasil, nos apropriamos de Signorelli (2011, p. 17), quando discorre sobre os diversos tipos de violência que acometem as mulheres, sinalizando que eles provocam múltiplas repercussões na saúde dessas mulheres. Portanto, a "violência física" compreende lesões e danos à integridade física das mulheres; a "violência psicológica" inclui humilhações, isolamento, desprezos e intimidações; a "violência patrimonial" se concretiza em roubo, retenção ou danos aos bens materiais, aos recursos e/ou documentos; já a "violência verbal" é caracterizada por xingamentos, ofensas e injúrias; e a "violência sexual" é caracterizada por relações sexuais forçadas ou práticas sexuais não consentidas. Dessa forma, com a instituição da Lei Maria da Penha, hoje se preconiza a punição para agressão física, enquadrando-se também a violência psicológica, sexual, patrimonial, além de proteção à mulher denunciante.

No decorrer da história e em diferentes culturas e sociedades as mulheres eram consideradas inferiores aos homens, no entanto, vimos que para cada ato ou tentativa de sujeição das mulheres em relação aos homens há a contrapartida da resistência feminina. A luta por direitos contribui para a consagração do atual aparato legal - Lei Maria da Penha, Lei n ${ }^{\circ} 11.340$, de 2016 -, que foi uma das grandes vitórias do movimento feminista. O nome homenageia a farmacêutica Maria da Penha Maia Fernandes, que ficou paraplégica após anos de violência doméstica. A lei visa prevenir a violência e punir de forma mais efetiva os homens - geralmente companheiros - agressores no âmbito familiar e doméstico, e contribuiu para a diminuição em $10 \%$ dos casos de assassinatos contra mulheres, segundo dados do IPEA de 2015.

No entanto, o Fórum de Segurança Pública divulgou recentemente o "Atlas da Violência de 2018”, destacando um dos casos que chocou o país e marcou o início do ano de 2018 no que tange à violência contra a mulher: o caso do assassinato da vereadora Marielle Franco. Através de uma comoção geral, o povo brasileiro viu seu nome virar símbolo de resistência de que a violência contra 
a mulher vem sendo desnaturalizada. Conforme os dados do Atlas, somente em 2016, 4.645 mulheres foram assassinadas no Brasil, com aumento de 6,4\% na taxa de feminicídio.

Ainda no tocante ao cenário da violência contra a mulher, o Fórum Brasileiro de Segurança Pública, no Atlas da Violência de 2019 (em que pese, esses dados são referentes ao ano de 2018) divulgou o forte crescimento dos homicídios femininos no Brasil em 2018, com cerca de 13 assassinatos por dia. Ao todo, 4.936 mulheres foram mortas, apresentando o maior número registrado desde 2017.

Face ao exposto e em decorrência dos números alarmantes da violência que acomete as mulheres, tendo por base os relatórios supracitados, considera-se o tema "violência contra as mulheres por parceiros íntimos" de extrema relevância no cenário atual, pois a todo momento deparamo-nos com notícias e casos que afetam as mulheres, indiferentemente de classes sociais, e, sobretudo, ligadas às questões como pobreza, gênero, preconceito, desigualdades socialmente produzidas, intolerância e as mais diferentes causas que atingem o público feminino.

O Atlas da Violência de 2019 revela que em 2017 mais de 221 mil mulheres procuraram as delegacias para registros dos casos de violência, dentre elas a lesão corporal. Esses números podem estar subestimados, pois dentre os fatores que desencorajam as vítimas a procurar ajuda podemos destacar a vergonha, o medo e a falta de conhecimento acerca das políticas e leis relacionadas à violência. Além disso, há aquelas que quando são obrigadas a procurar o serviço de saúde devido a alguma lesão, por medo, mentem e silenciam o caso. Portanto, o desenvolvimento de propostas alternativas de apoio, como a de uma plataforma online, anônima, pode significar em última instância, salvar vidas de mulheres.

Para Signorelli (2011), as questões de gênero, junto com as desigualdades sociais, são elementos essenciais na (re)produção de violências e, portanto, merecem atenção para o fator desigualdade (social e de gênero) que se manifesta no seio das assimetrias de poder.

Nesse cenário, cunhada numa dimensão emancipatória e política, pretende-se com a proposta de uma "Plataforma on-line para apoio à tomada de decisão e planejamento de segurança para mulheres vivendo com violência doméstica no Brasil” materializar a adaptação de plataformas já existentes ao contexto social brasileiro, bem como implementar práticas inovadoras de enfrentamento à violência contra a mulher por meio do uso da tecnologia, ou seja, um meio de contribuir com a redução do conflito decisório, no apoio à tomada de decisão e no planejamento de segurança.

Portanto, ao propor um instrumento de apoio à tomada de decisão, vislumbra-se criar novas possibilidades para romper o ciclo vicioso da relação abusiva de violência que acomete grande parcela 
das mulheres no Brasil como mecanismo de construção coletiva de uma política de consagração de direitos humanos, constituindo-se em uma prática social, pedagógica, interativa e dialógica, numa perspectiva interdisciplinar em favor da equidade e da justiça social em atenção às mulheres em situação de VPI.

\section{Considerações finais}

Este artigo apresentou as principais bases teórico-metodológicas de uma proposta já testada e avaliada internacionalmente, que visa apoiar mulheres vivendo em situação de violência doméstica a tomar a decisão de largar seu relacionamento abusivo. Com base na pesquisa de campo que vem sendo desenvolvida com profissionais e mulheres atendidas na Casa da Mulher Brasileira de Curitiba, foi possível enumerar aspectos preliminares relevantes para conceber a versão brasileira da plataforma, levando-se em conta os desafios, potencialidades e sugestões. A maioria das participantes considerou que a proposta é viável, contudo, é preciso planejar medidas de segurança quanto ao uso, levando em conta que algumas mulheres sofrem monitoramento constante feito pelo agressor. Adicionalmente, é preciso interligar o aplicativo com a rede de proteção e serviços existentes no Brasil.

A partir das sugestões elencadas, a próxima etapa consistirá no desenvolvimento da plataforma, em conjunto com as participantes, com sua constante avaliação quanto ao design, funcionalidades, utilização, informações, etc. Esta pesquisa almeja subsidiar a materialização de um recurso tecnológico de fácil acesso, disponível gratuitamente para redução da violência contra mulheres brasileiras. Tal plataforma on-line se configurará como uma ferramenta sustentável de apoio à tomada de decisão para redução de conflito e incentivo à denúncia, disponível e acessível de qualquer local para que a mulher sinta-se apoiada em suas decisões e exerça um papel ativo no que tange a avaliar os riscos da violência e conexão com os serviços de apoio presencial.

\section{Referências}

ACOSTA, D. F.; GOMES, V. L. O.; FONSECA, A. D.; GOMES, G. C. Violência contra a mulher por parceiro íntimo: (in) visibilidade do problema. Texto Contexto Enferm., Florianópolis, v. 24, n. 1, jan./mar., p. 121-127, 2015. 
AUAD, D. Feminismo: que história é essa? Rio de Janeiro: DP\&A, 2003.

BARDIN, L. Análise de conteúdo. Lisboa: Edições 70, 1995.

BEAUVOIR, S. O segundo sexo. Fatos e mitos. 4. ed. São Paulo: Difusão Européia do livro, 1949.

BRASIL. Lei Federal no 11. 340, de 07 de agosto de 2006. Dispõe sobre a Lei Maria da Penha e dá outras providências. Brasília: Ministério da Justiça, 2006. Disponível em http://www.planalto.gov.br/ccivil_03/_ato2004-2006/2006/lei/111340.htm. Acesso em: 15 set. 2019.

CLEMENTE, A. J; MARTINEZ; I. N; HOFFMANN-HOROCHOVSKI, M.T. Violência contra a mulher em São Borja (RS): apontamentos para o debate. In: PINTO, M.; GOMES, A.Q. (Orgs.). Políticas públicas, cultura e dinâmicas sociais na fronteira Brasil e Argentina. Jaguarão, RS:

CLAEC, 2018. p. 42-62.

DUTTON, M. A. Empowering and healing the battered woman: a model assessment and intervention. New York: Springer, 1992.

EDEN, K. B. et al. Use of online safety decision aid by abused women: effect on decisional conflict in a randomized controlled trial. Am J Prev Med, v. 48, n. 4, p. 372-83, apr., 2015. ISSN 1873-2607. Disponível em: https://www.ncbi.nlm.nih.gov/pubmed/25547929. Acesso em: 01 set. 2019.

FORD-GILBOE, M. et al. A tailored online safety and health intervention for women experiencing intimate partner violence: the iCAN Plan 4 Safety randomized controlled trial protocol. BMC Public Health, 2017. Disponível em < https://www.ncbi.nlm.nih.gov/pmc/articles/PMC5360053/>, acesso em 19/12/2019.

TARZIA, L. et al. I-Decide: an online intervention drawing on the psychosocial readiness model for women experiencing domestic violence. Women's Health Issues, 2016. Disponível em < https://www.ncbi.nlm.nih.gov/pubmed/26362841>, acesso em 19/12/2019.

HEGARTY, K. et al. An online healthy relationship tool and safety decision aid for women experiencing intimate partner violence (I-DECIDE): a randomised controlled trial. The Lancet Public Health, 2019. Disponível em < https://www.thelancet.com/journals/lanpub/article/PIIS24682667(19)30079-9/fulltext?rss=yes >, acesso em 19/12/2019.

HEGARTY, K. et al. Protocol for a randomised controlled trial of a web-based healthy relationship tool and safety decision aid for women experiencing domestic violence (I-DECIDE). BMC Public Health, v. 15, p. 736, aug. 2015. ISSN 1471-2458. Disponível em: https://www.ncbi.nlm.nih.gov/pubmed/26231225. Acesso em: 15 ago. 2019.

INSTITUTO DE PESQUISA APLICADA (IPEA). FÓRUM BARSILEIRO DE SEGURANÇA PÚBLICA. Atlas da violência 2018. Apresenta indicadores para melhor compreender o processo de acentuada violência no país. Rio de Janeiro, 2018. Disponível em: http://www.ipea.gov.br/portal/index.php?option=com_content \&view=article\&id=33410\&Itemid=4 32. Acesso em: 16 set. 2019. 
INSTITUTO DE PESQUISA APLICADA (IPEA). FÓRUM BARSILEIRO DE SEGURANÇA PÚBLICA. Atlas da violência 2018. Apresenta o retrato da violência nos municípios brasileiros. Brasília, 2019. Disponível em: http://www.forumseguranca.org.br/wpcontent/uploads/2019/06/Atlas-da-Violencia-2019_05jun_vers\%C3\%A3o-coletiva.pdf.

Acesso em: 16 set. 2019.

JAFFE, P. G.; BAKER, L. L. et al. (Ed.). Protecting children from domestic violence: strategies for community intervention. New York: Guilford Press, 2004. p. 89-100.

KOZIOL-MCLAIN, J. et al. Participant recruitment and engagement in automated e-health trial registration: challenges and opportunities for recruiting women who experience violence. J. Med. Internet Res., v. 18, n. 10, p. e281, oct. 2016. ISSN 1438-8871. Disponível em: https://www.ncbi.nlm.nih.gov/pubmed/27780796. Acesso em: 18 jul. 2019.

KOZIOL-MCLAIN, J. et al. A web-based intervention for abused women: the New Zealand isafe randomised controlled trial protocol. BMC Public Health, v. 15, p. 56, jna. 2015. ISSN 1471-2458. Disponível em: https://www.ncbi.nlm.nih.gov/pubmed/25637195.

KRUG, E. G.; DAHLBERG, L. L; MERCY, J. A.; ZWI, A. B.; LOZANO, R. Relatório mundial sobre violência e saúde. Genebra: Organização Mundial da Saúde, 2002.

LIAMPUTTONG, P.; EZZY, D. Qualitative Research Methods. 2a. Ed. Melbourne: Oxford, 2005.

MINAYO, M. C. S. Pesquisa social: teoria, método e criatividade. Petrópolis: Vozes, 2002.

PEDRO, J. M. Traduzindo o debate: o uso da categoria gênero na pesquisa histórica. História, São Paulo, v. 24, n. 1, p. 77-98, 2005.

SIGNORELLI, M. C. Mudaram as estações...nada mudou: profissionais do Sistema Único de Saúde e mulheres vítimas de violência doméstica no litoral paranaense. 194f. Tese (Doutorado) Programa de Pós-graduação em Saúde Coletiva, Universidade Federal de São Paulo, 2011.

TRIPP, D. Pesquisa-ação: uma introdução metodológica. Educação e Pesquisa, São Paulo, v. 31, n. 3, p. 443-466, set./dez. 2005.

Artigo recebido em 03/12/2019. Aceito para publicação em 19/12/2019. 